\title{
A Bilevel Programming Approach to Assembly Job Shop Scheduling
}

\author{
George Q. Huang ${ }^{1}$, Haili Lu $^{2}$ \\ ${ }^{1}$ Department of industrial \& manufacturing systems engineering, Hong Kong University, Hong Kong \\ (gqhuang@hku.hk) \\ ${ }^{2}$ Department of industrial \& manufacturing systems engineering, Hong Kong University,Hong Kong
} (lvhaili@hku.hk)

\begin{abstract}
A bilevel programming approach for assembly job shop scheduling is proposed. Two levels of decision makers are identified in the model. The first level is the project manager and the second level is the shop floor manager. The first level aims to minimize the earliness and tardiness of completed jobs. The second level aims to minimize the average shop floor throughput time. Because their aims may conflict, these levels optimize their own objectives based on a non-cooperative game playing process. Their decision variables are denoted by order release mechanisms and dispatching rules respectively. Using a simulation approach, this paper identifies the best choice for the project manager under different job shop utilization levels. The research findings can provide managerial guidance to the project manager as which order release mechanisms to use in order to optimize his objective.
\end{abstract}

Keywords: bilevel programming, assembly job shop scheduling, order review and release, dispatching rules

\section{INTRODUCTION}

This paper is motivated by the research and practice in a Mold and Die Manufacturing (MDM) company in Guangdong province, China. This research involves two related areas in the literature. They are assembly job shop scheduling, or job shop scheduling with assembly operations [1] and bilevel [2] decision structure.

Assembly job shop scheduling is characterized by an assembly operation. In mold and die manufacturing, final products are assembled by several components. The assembly of a final product can only be conducted after all the components are finished. The components of a final product are in turn processed by different machines according to a predetermined process plan.

Bilevel decision structure is another characteristic of this research. As in most companies, a hierarchy of production planning and scheduling exists. We have studied the company's organization structure and found that two levels of decision makers exist in the company. The higher level is the project manager and the lower level is the shop floor manager. The two levels have different objectives and decision rights (variables). The upper level concerns about the due date performance while the lower level cares about reduction of WIP inventory. It is noteworthy mentioning that the two objectives are not always compatible. Thus the bilevel scheduling problem takes on the features of a non-cooperative game playing process. It can be expected that if the shop floor manager works in collaboration with the project manager, and seeks no

978-1-4244-4136-5/09/\$25.00 @2009 IEEE sub-optimization of his own objective, the objective of the project manager should be better fulfilled. Under the bilevel structure, the shop floor manager has some freedom in optimizing his objective of WIP inventory. The freedom of decision inevitably causes deterioration of the project manager. This situation is unfavorable from the project manager's point of view. After the project manager authorizes the release of orders into the shop floor, the shop floor manager takes full control of the production process. It is difficult and almost impossible for the project manager to oversee the everyday production process in the shop floor. However, the project manager can exert his influence by controlling the timing of release of orders to the shop floor. By choosing different order release mechanisms, the project manager can improve the due date performance of orders. In order to better optimize the objective of the project manager, we have established a bilevel scheduling model and conducted a simulation study to find out suitable control policies for the project manager in different utilization levels.

\section{LITREATURE REVIEW}

\subsection{Assembly job shop scheduling}

Assembly job shop scheduling is different from string type job shop scheduling. The main difference is that assembly job shop scheduling involves not only the string type scheduling decision, but also coordination of the assembly operations. Considerable amount of research has been done on assembly job shop scheduling [3-8]. All of the reviewed literature focuses on different dispatching rules using a simulation approach. Except the common dispatching rules which are used in string type job shop, some rules have been specifically developed for assembly job shop scheduling. For 
example, NUP[1] and TWKR[9] are two rules which prove efficient in assembly job shop scheduling and will be included in our later experiment.

\subsection{Bilevel programming}

Bracken and McGill [2] proposed a formulation for bilevel programming. While it is Candler and Norton [10] who explicitly used multilevel and bilevel programming to denote this decision structure.

Bilevel programming assumes a sequential decision process. In bilevel programming, each decision maker has his own objective and control variables. The higher level decision maker sets his decision variables first, and reveals it to the lower level decision maker. The lower level will set his decision variable in order to optimize his objective based on the decision of the higher level. This sequential decision process is repeated until the higher level has tried all possible decision variables. The aim of the higher level is to choose an appropriate decision variable such that his objective can be optimized.

The concept of bilevel programming has been successfully applied in a variety of real life problems. Example papers in production planning and scheduling include [11-13].

\section{MODEL DESCRIPTION}

\subsection{The job shop model}

The model is based on a simulated assembly job shop similar to those assumed in several previous studies[14, 15]. The simulation is written in Tecnomatix Plant Simulation version 8.1.

In the jobs shop, six machines are deployed. Jobs arrive according to a Poisson process at a mean rate of 3.47 , 3.26 and 3.10 units per hour, producing utilization rates of $80 \%, 85 \%$ and $90 \%$ respectively. A job is assembled by 2 to 6 different parts, with the number of parts uniformly distributed. A part requires processing operations on 2 to 6 machines, with the number of total operations uniformly distributed. Processing time of an operation on any machine is exponentially distributed with a mean of 1 hour. Each machine has equal possibility of being the first and last in the processing sequence. Revisit to a machine is only allowed after at least one different operation.

The due date of a job is set using the TWKCP (total work content on the critical path) rule[16]. Specifically, after the arrival of a job, the due date is set by adding to the arriving time of the job a multiple of the largest total processing time of all components. The multiplier is a uniform random number sampled from interval $(5,13)$.

\subsection{Performance criteria}

As stated in the introduction section, two levels of decision makers have different objectives. The project manager at the high level aims at improving due date performance of completed jobs, while the shop floor manager at the lower level aims at optimizing work in process inventory. In order to reflect their corresponding concerns, we use mean absolute deviation ( $M A D$ ) of all completed jobs as the performance measure of the project manager, and use average shop floor throughput time ( $S F T T$ ) as the performance measure of the shop floor manager. The average throughput time SFTT is used as an effective approximate to the average shop floor inventory.

Firstly, the absolute deviation for a job $i$ is defined as follows:

$$
A D_{i}=\max \left(0,\left|C_{i}-D_{i}\right|\right)
$$

$D_{i}:$ Due date of job $i$

$C_{i}$ : Completion time of job $i$

The mean absolute deviation is calculated by dividing the total absolute deviations by the number of jobs.

$$
M A D=\frac{1}{n} \sum_{i=1}^{n} A D_{i}
$$

$n:$ Number of jobs

The measure $M A D$ includes both tardy and early information of job completions. As shown below, $A D_{i}$ can be decomposed into two parts:

$$
\begin{aligned}
& A D_{i}=\max \left(0,\left|C_{i}-D_{i}\right|\right) \\
& =\max \left(0, C_{i}-D_{i}\right)+\max \left(0, D_{i}-C_{i}\right)
\end{aligned}
$$

The first part in equation (3) stands for job tardiness and the second part stands for the job earliness. Thus the MAD measure is comparable to an alternate measure of earliness and tardiness cost. However, no information about the relative cost of earliness and tardiness is included in equation (3).

The second performance criterion is the average shop floor throughput time (SFTT). SFTT is defined as the time the order is released to the shop floor to the time the final product is due and delivered, as shown below.

$$
S F T T_{i}=\frac{1}{N O P_{i}} \sum_{j=1}^{N O P_{i}}\left(C_{i}-R_{i j}\right)
$$

$N O P_{i}$ : Number of components for product $i$

$R_{i j}$ : Release time of component $j$ of order $i$ into the shop floor

The average shop floor throughput time $S F T T$ is then calculated as follows.

$$
S F T T=\frac{1}{n} \sum_{i=1}^{n} S F T T_{i}
$$

\subsection{Decision variables}

\subsubsection{Decision variables of the project manager}

The project manager is at the higher level and controls 
the release of orders to the shop floor. A job that arrives is not necessarily released to the shop floor immediately. The project manager will determine the release time of each component by using some order release mechanisms. Three order release mechanisms are considered in the simulation model. All of the release mechanisms have appeared in earlier researches. A detailed description can be found in related literature.

Set of order release mechanisms ( $R E L)$ :

- IMM(Immediate release) [17]:

This method releases a job into the shop floor immediately when a job arrives.

- BIL(Backward infinite loading) [15]:

This method calculated the release time of each part of a job using the following equation.

$R_{i j}=D_{i}-k_{1} \times n_{i j}$

$k_{1}$ : Planning factor to be determined.

$n_{i j}$ : Number of operations needed for processing component $j$ of product $i$

The release date is calculated at the beginning of each planning period. If the release time is equal to or earlier than the current time, the component is released to the shop floor. If not, the component will be sent to a pre-shop pool waiting for release in later review periods.

- MIL(Modified backward infinite loading) [14]:

MIL is similar to MIL, but it considers the shop information when determining the release date of a particular part.

$R_{i j}=D_{i}-k_{1} \times n_{i j}-k_{2} \times J I Q_{i j}$

$k_{1}$ : Planning factor to be determined

$k_{2}$ : Planning factor to be determined

$J I Q_{i j}$ : Number of jobs waiting in queues of machines that this component will visit

The release date is calculated at the beginning of each planning period. If the calculated release date is equal to or earlier than the present, the component is released to the shop floor.

\subsubsection{Decision variables of the shop floor manager}

The shop floor manager takes control of the jobs after they are released to the shop floor. Their decision is to decide which dispatching rule to use. That is, when a machine becomes available, the dispatching rule determines which job in the waiting queues will be processed next. The dispatching rules are illustrated as below.

Set of dispatching rules ( $D I S)$ :

- FCFS (First comes first serviced): Jobs that arrive at a machine earliest will be processed first.

- FASFS (First arrives into system first serviced): Jobs which are released into the shop floor earliest will be processed first.
- SPT (Shortest processing time): Jobs with the shortest processing time will be processed first.

- NUP (Number of unfinished parts) [1]: Jobs with the fewest number of unfinished components will be processed first.

- TWKR (Total work remaining) [9]: Jobs with the least amount of remaining processing time will be processed first.

- JDD (Job due date): Jobs with the earliest due date will be processed first.

\subsection{The bilevel decision model}

In the simulation, we assume machine utilization levels and job arrival rates remain constant, and such information is known by both the project manager and the shop floor manager. In order to mimic the decision process, we generate the test data using information about job arrival patterns, BOM structure, job routing and processing times etc., as stated in section 3.1. For each set of test data, we assume the project manager and shop floor manager determines their variables in the following way. Firstly, the project manager tries a release method and reveals it to the shop floor manager. After knowing the decision of the project manager, the shop floor manager will try every possible dispatching rule to minimize the average shop floor throughput time measure (SFTT). After the project manager tests all release methods, he will choose the release method with the minimum mean absolute deviation (MAD).

The decision structure can be illustrated as follows.

Parameters:

$R E L: \quad$ Set of order release methods

DIS : $\quad$ Set of dispatching rules

Variables:

rel : Release method used by the project manager, rel $\in R E L$

dis : Dispatching rule used by the shop floor manager, dis $\in$ DIS

The bilevel model:

Min $M A D$

With respect to $r e l \in R E L$

s.t. $d i s \in D I S$

Where $d$ solves

Min SFTT

s.t. Operation precedence constraints Assembly constraints

\section{EXPERIMENT SETTINGS}

The experiments consist of a full factorial study of the release mechanisms and dispatching rules listed above. That is, we test all the policy combinations of ORR and dispatching rules. Analysis of results is then conducted to find the best release rule for the project manager.

Essentially, the experiment consists of two phases. In the first phase, a series of trial runs are conducted to 
search for appropriate values for the parameters used in BIL and MIL order release mechanisms. The parameters are set to produce an as small value of MAD as possible for each combination of order release and dispatching rule. The parameter search process is similar to that used by Ragarts etc[14]. After all parameters are fixed, the second phase experiments are conducted. Five replications are conducted for each policy combination. In order to ensure that all the policy combinations are subject to the same test data, "common random number" technique is used[18].

\section{RESULTS}

The experiment is first conducted for utilization level of $85 \%$. The result is shown in table 1 . The values in the brackets in the runs with BIL and MIL are the values of planning factor $k_{1}$ and $k_{2}$. Using the bilevel decision structure defined in section 3.4 , we can find the best solution in the following way. When the project manager uses IMM, the shop floor manager will use the dispatching rule which minimizes the SFTT. In this case, he will choose TWKR, which results in a minimum SFTT of 28.21, and a MAD value of 37.43, as shown in run 5 . When the project manager uses BIL, the shop floor manager will use TWKR as the dispatching rule, which results in a minimum SFTT of 27.87, and a MAD value of 14.92 , as shown in run 12 . When the project manager uses MIL, the shop floor manager will use NUP as the dispatching rule, which result in a minimum SFTT of 25.15, and a MAD value of 14.06, as shown in run 18. Using the above information, the project manager should use MIL policy. This would result in a MAD value of 14.06 as shown in run 18 .

Besides the utilization level of $85 \%$, experiments of lower and higher utilization levels are also conducted.
The results are shown in table 2 and 3. Analysis of results is similar for $80 \%$ utilization level. We can see from table 2 that the best decision for the project manager is to use MIL. The rational choice for the shop floor manager will be TWKR. This results in a MAD of 10.56 as shown in run 19 . When $90 \%$ utilization is used, we can see from table 3 that the best decision for the project manager is MIL. The best decision for the shop floor manager is TWKR. The resulting MAD is 16.63 as shown in run 19.

Comparing the analysis for the three utilization levels, three conclusions can be made. Firstly, TWKR or NUP performs consistently well in minimizing SFTT, regardless of the release rules used. Secondly, MIL release seems to perform consistently better than BIL release regardless of the dispatching rules used. Thirdly, the decisions of the project manager and the shop floor manager seem to be insensitive to the utilization levels used. Under the bilevel decision structure, the project manager's best choice should be MIL, and the shop floor manager's best choice should be NUP or TWKR, under all the three utilization levels.

We can also see that the bilevel decision structure brings deterioration to the objective function values of the project manager. In table 1 , the minimum possible MAD value is 12.72 in run 13 , when BIL is used as the release rule and JDD is used as the dispatching rule. Under the bilevel decision structure, the best MAD value is 14.06 in run 18 , slightly worse than the value of 12.72 . The deterioration of MAD value is due to the uncooperative decision of the shop floor manager.

Table 1 Experiment result for $85 \%$ utilization

\begin{tabular}{|c|c|c|c|c|c|}
\hline Experiment & Parameter combination & Average tardiness & Average earliness & MAD & SFTT \\
\hline 1 & IMM-FCFS & 8.05 & 22.42 & 30.47 & 44.52 \\
\hline 2 & IMM-FASFS & 3.67 & 26.64 & 30.31 & 35.91 \\
\hline 3 & IMM-SPT & 6.42 & 31.21 & 37.63 & 34.11 \\
\hline 4 & IMM-NUP & 4.79 & 33.55 & 38.34 & 30.13 \\
\hline 5 & IMM-TWKR & 3.38 & 34.05 & 37.43 & 28.21 \\
\hline 6 & IMM-JDD & 0.99 & 26.96 & 27.95 & 32.92 \\
\hline $7(11)$ & BIL-FCFS & 11.14 & 5.70 & 16.84 & 41.86 \\
\hline $8(11)$ & BIL-FASFS & 8.96 & 5.67 & 14.63 & 39.72 \\
\hline $9(7)$ & BIL-SPT & 14.26 & 4.40 & 18.66 & 33.46 \\
\hline $10(7)$ & BIL-NUP & 10.91 & 4.96 & 15.87 & 29.55 \\
\hline $11(7)$ & BIL-TWKR & 9.60 & 5.33 & 14.92 & 27.87 \\
\hline $12(9)$ & BIL-JDD & 7.73 & 4.99 & 12.72 & 33.27 \\
\hline $13(12-1)$ & MIL-FCFS & 10.67 & 5.22 & 15.89 & 33.32 \\
\hline $14(12-1)$ & MIL-FASFS & 9.36 & 5.07 & 14.43 & 32.42 \\
\hline $15(10-2)$ & MIL-SPT & 10.07 & 4.29 & 14.36 & 25.76 \\
\hline $16(9-0.8)$ & MIL-NUP & 9.90 & 4.16 & 14.06 & 25.16 \\
\hline $17(9-0.5)$ & MIL-TWKR & 7.46 & 6.05 & 13.51 & 25.39 \\
\hline $18(9-0.5)$ & MIL-JDD & 10.41 & 3.50 & 13.91 & 29.97 \\
\hline
\end{tabular}


Table 2 Experiment result for $80 \%$ utilization

\begin{tabular}{|c|c|c|c|c|c|}
\hline Experiment & Parameter combination & Average tardiness & Average earliness & MAD & SFTT \\
\hline 1 & IMM-FCFS & 3.88 & 27.39 & 31.27 & 35.38 \\
\hline 2 & IMM-FASFS & 1.81 & 31.28 & 33.08 & 29.42 \\
\hline 3 & IMM-SPT & 3.04 & 34.31 & 37.36 & 27.62 \\
\hline 4 & IMM-NUP & 2.62 & 36.10 & 38.72 & 25.41 \\
\hline 5 & IMM-TWKR & 1.64 & 36.61 & 38.26 & 23.92 \\
\hline 6 & IMM-JDD & 0.30 & 31.63 & 31.94 & 27.56 \\
\hline $7(9)$ & BIL-FCFS & 7.91 & 4.91 & 12.82 & 33.52 \\
\hline $8(9)$ & BIL-FASFS & 7.09 & 4.70 & 11.78 & 32.91 \\
\hline $9(6.3)$ & BIL-SPT & 9.94 & 3.69 & 13.63 & 27.23 \\
\hline $10(5.7)$ & BIL-NUP & 9.09 & 2.95 & 12.05 & 24.79 \\
\hline $11(6.5)$ & BIL-TWKR & 6.77 & 4.75 & 11.52 & 23.73 \\
\hline $12(8)$ & BIL-JDD & 5.27 & 5.00 & 10.27 & 27.45 \\
\hline $13(10-0.5)$ & MIL-FCFS & 7.09 & 5.52 & 12.62 & 29.97 \\
\hline $14(10-0.5)$ & MIL-FASFS & 6.36 & 5.27 & 11.64 & 29.62 \\
\hline $15(9-1.5)$ & MIL-SPT & 6.48 & 5.02 & 11.50 & 22.93 \\
\hline $16(8-0.5)$ & MIL-NUP & 6.14 & 4.99 & 11.13 & 22.54 \\
\hline $17(7-0.5)$ & MIL-TWKR & 7.19 & 3.38 & 10.56 & 21.86 \\
\hline $18(9-0.2)$ & MIL-JDD & 4.29 & 6.40 & 10.70 & 26.17 \\
\hline
\end{tabular}

Table 3 Experiment result for $90 \%$ utilization

\begin{tabular}{|c|c|c|c|c|c|}
\hline Experiment & Parameter combination & Average tardiness & Average earliness & MAD & SFTT \\
\hline 1 & IMM-FCFS & 15.22 & 17.16 & 32.38 & 56.95 \\
\hline 2 & IMM-FASFS & 7.08 & 21.50 & 28.57 & 44.47 \\
\hline 3 & IMM-SPT & 11.68 & 28.33 & 40.01 & 42.24 \\
\hline 4 & IMM-NUP & 8.70 & 31.17 & 39.87 & 36.42 \\
\hline 5 & IMM-TWKR & 6.29 & 31.90 & 38.19 & 33.28 \\
\hline 6 & IMM-JDD & 2.66 & 21.00 & 23.66 & 40.54 \\
\hline $7(12)$ & BIL-FCFS & 17.68 & 4.66 & 22.34 & 51.96 \\
\hline $8(13)$ & BIL-FASFS & 12.65 & 5.62 & 18.27 & 48.29 \\
\hline $9(7)$ & BIL-SPT & 21.37 & 3.82 & 25.19 & 41.12 \\
\hline $10(8)$ & BIL-NUP & 14.32 & 6.48 & 20.80 & 35.03 \\
\hline $11(7)$ & BIL-TWKR & 14.06 & 4.87 & 18.93 & 32.77 \\
\hline $12(11)$ & BIL-JDD & 10.30 & 5.85 & 16.15 & 40.85 \\
\hline $13(14-1)$ & MIL-FCFS & 15.38 & 5.06 & 20.44 & 40.50 \\
\hline $14(12-0.2)$ & MIL-FASFS & 13.94 & 4.21 & 18.15 & 45.32 \\
\hline $15(12-2)$ & MIL-SPT & 12.56 & 5.87 & 18.44 & 30.77 \\
\hline $16(11-0.7)$ & MIL-NUP & 11.24 & 6.17 & 17.41 & 29.72 \\
\hline $17(11-1)$ & MIL-TWKR & 11.33 & 5.29 & 16.63 & 28.18 \\
\hline $18(13-0.1)$ & MIL-JDD & 8.07 & 8.64 & 16.71 & 40.68 \\
\hline
\end{tabular}

※ For experiments using BIL, the values in the brackets are estimated values for $k_{1}$

※ For experiments using MIL, values in the brackets are estimated values for $k_{1}$ and $k_{2}$ respectively. 
Similar observations can be made from table 2 and 3 . However, it is interesting to find that the deterioration is not great. This may be due to the great advantage of the NUP and TWKR dispatching rule. For assembly job shop scheduling, these two rules not only produces small SFTT but also produces small MAD value.

\section{CONCLUSION AND FUTURE RESEARCH DIERECTIONS}

It should be clarified that, although BIL and MIL is treated as fixed parameter settings in our simulation and other literature, there is still a need to find appropriate value of the planning factor $k_{1}$ and $k_{2}$. Although the factors are set to produce an as small value of MAD as possible for each combination of order release and dispatching rule, we can not guarantee that the values found are optimal.

The research in this paper only involves BIL and MIL release mechanisms. However, many other release mechanisms can be found in the literature such as load oriented order review and release[19]. Further research can be conducted to include more of the available release mechanisms.

\section{REFERENCES}

[1] W. L. Maxwell, "Priority dispatching and assembly operations via job shop," Report Rm-5370-PR, Rand Corporation, CA, 1969, 1969.

[2] J. Bracken, and J. T. McGill, "Mathematical programs with optimization problems in the constraints," Operations Research, vol. 21, no. 1, pp. 37-44, 1973.

[3] P. R. Philipoom, R. E. Markland, and T. D. Fry, "Sequencing rules, progress milestones and product structure in a multistage job shop," Journal of Operations Management, vol. 8, no. 3, pp. 209-229, 1989.

[4] S. Thiagarajan, and C. Rajendran, "Scheduling in dynamic assembly job-shops to minimize the sum of weighted earliness, weighted tardiness and weighted flowtime of jobs," Computers \& Industrial Engineering, vol. 49, no. 4, pp. 463-503, 2005.

[5] K. Natarajan, K. Mohanasundaram, B. Babu et al., "Performance evaluation of priority dispatching rules in multi-level assembly job shops with jobs having weights for flowtime and tardiness," The International Journal of Advanced Manufacturing Technology, vol. 31, no. 7, pp. 751-761, 2007.

[6] N. R. Adam, J. W. M. Bertrand, and J. Surkis, "Priority assignment procedures in multi-level assembly job shops," IIE Transactions, vol. 19, no. 3, pp. 317 - 328, 1987.

[7] M. Reeja, and C. Rajendran, "Dispatching rules for scheduling in assembly jobshops-Part 1," International Journal of Production Research, vol. 38, no. 9, pp. 2051-2066, 2000.

[8] M. Reeja, and C. Rajendran, "Dispatching rules for scheduling in assembly jobshops- Part 2," International Journal of Production Research, vol. 38, no. 10, pp. 2349-2360, 2000.

[9] G. Siegel, "An investigation of job shop scheduling for jobs with assembly constraints," Unpublished PhD dissertation, Cornell University, 1971.

[10] W. Candler, and R. Norton, "Multilevel programming," Technical report 20, World bank development research centre, washington D..C, 1977.

[11] Z. Lukac, K. Soric, and V. V. Rosenzweig, "Production planning problem with sequence dependent setups as a bilevel programming problem," European Journal of Operational Research, vol. 187, no. 3, pp. 1504-1512, 2008.

[12] K. K. John, and W. Wei, "Bilevel programming applied to the flow shop scheduling problem," Comput. Oper. Res., vol. 23, no. 5, pp. 443-451, 1996.

[13] M. G. Nicholls, "Aluminum production modeling-A nonlinear bilevel programming approach," Operations Research, vol. 43, no. 2, pp. 11, 1995.

[14] G. Ragatz, and V. Mabert, "An evaluation of order release mechanisms in a job-shop environment," Decision Sciences, vol. 19, no. 1, pp. 167-189, 1988.

[15] I. Ahmed, and W. Fisher, "Due date assignment, job order release, and sequencing interaction in sob shop scheduling," Decision Sciences, vol. 23, no. 3, pp. 633-647, 1992.

[16] A. Hatchuel, D. Saidi-Kabeche, and J. Sardas, "Towards a new planning and scheduling approach for multistage production systems," International Journal of Production Research, vol. 35, no. 3, pp. 867-886, 1997.

[17] I. Ahmed, and W. Fisher, "Due date assignment, job rrder release, and sequencing interaction in sob shop scheduling," Decision Sciences, vol. 23, no. 3, pp. 633-647, 1992.

[18] N. Adam, J. Bertrand, and J. Surkis, "Priority Assignment Procedures in Multi-Level Assembly Job Shops," IIE Transactions, vol. 19, no. 3, pp. 317-328, 1987.

[19] W. Bechte, "Load-oriented manufacturing control just-in-time production for job shops," Production Planning \& Control, vol. 5, no. 3, pp. 292-307, 1994. 